\title{
Genetic Linkage of Type VII Collagen (COL7A1) to Dominant Dystrophic Epidermolysis Bullosa in Families with Abnormal Anchoring Fibrils
}

\author{
Markku Ryynänen, Jaana Ryynănen, Stephan Sollberg, Renato V. lozzo, Robert G. Knowlton, and Jouni Uitto \\ Departments of Dermatology, Biochemistry and Molecular Biology, and Pathology and Cell Biology, Jefferson Medical College; and \\ Section of Molecular Dermatology, Jefferson Institute of Molecular Medicine, Thomas Jefferson University, Philadelphia, Pennsylvania \\ 19107
}

\begin{abstract}
Epidermolysis bullosa (EB) is a group of genodermatoses characterized by the fragility of skin. Previous studies on the dystrophic (scarring) forms of EB have suggested abnormalities in anchoring fibrils, morphologically recognizable attachment structures that provide stability to the association of the cutaneous basement membrane to the underlying dermis. Since type VII collagen is the major component of the anchoring fibrils, we examined the genetic linkage of dominant dystrophic EB (EBDD) and the type VII collagen gene (COL7A1) locus, which we have recently mapped to chromosome $3 p$, in three large kindreds with abnormal anchoring fibrils. Strong genetic linkage of EBDD and COL7A1 loci was demonstrated with the maximum logarithm of odds (LOD) score of 8.77 at $\hat{\theta}=0$. This linkage was further confirmed with two additional markers in this region of the short arm of chromosome 3, and these analyses allowed further refinement of the map locus of COL7A1. Since there were no recombinants between the COL7A1 and EBDD loci, our findings suggest that type VII collagen is the candidate gene that may harbor the mutations responsible for the EB phenotype in these three families. (J. Clin. Invest. 1992.89:974-980.) Key words: basement membrane zone • bullous dermatosis • genetic skin diseases
\end{abstract}

\section{Introduction}

Epidermolysis bullosa (EB) ${ }^{1}$ is a heterogenous group of mechano-bullous diseases characterized by easy blistering as a result of minor trauma. EB can be divided into three major categories on the basis of the level of tissue separation within the cutaneous basement membrane zone at the dermal-epidermal junction. In the simplex (nonscarring) forms, the blisters develop within the epidermis at the level of the basal layer of keratinocytes; in the junctional forms, the tissue separation occurs within the basement membrane at the level of the lamina lucida; and in the dystrophic (scarring) forms, the blister formation occurs below the basement membrane within the

\section{Address correspondence to Jouni Uitto, M. D., Ph. D., Thomas Jeffer- son University, Department of Dermatology, Bluemle Life Sciences Building, 233 South 10th Street, Suite 450, Philadelphia, PA 19107. \\ Received for publication 19 August 1991 and in revised form 24 October 1991.}

1. Abbreviations used in this paper: COL7A1, type VII collagen gene; EB, epidermolysis bullosa; EBDD, dominant dystrophic EB; EBS, simplex EB; LOD, logarithm of odds; RFLP, restriction fragment length polymorphism.

J. Clin. Invest.

(c) The American Society for Clinical Investigation, Inc.

0021-9738/92/03/0974/07 \$2.00

Volume 89, March 1992, 974-980 papillary dermis. The inheritance pattern can be either autosomal dominant or autosomal recessive (for reviews see references 1-3).

The patients with dystrophic EB have the most severe form of this condition. In autosomal recessive EB, the tissue separation can result from minor trauma, and the healing occurs with either atrophic or hypertophic scarring. In somewhat less severe, dominantly inherited forms of dystrophic EB (EBDD), the clinical manifestations are primarily present at the pressure points, hands, and feet; and the cutaneous changes are often associated with characteristic nail dystrophy. A diagnostic ultrastructural feature of the dystrophic forms of EB is altered morphology and marked reduction in the number of anchoring fibrils, attachment complexes at the dermal side of the cutaneous basement membrane zone (4-6). In severe recessive dystrophic forms of EB, the anchoring fibrils can be entirely absent (7), whereas in EBDD some anchoring fibrils may be present, but they are often hypoplastic and appear abnormal (4).

Type VII collagen is the major component of anchoring fibrils $(8,9)$. This collagen is a homotrimer, $[\alpha 1(\mathrm{VII})]_{3}$, and the individual molecules have been suggested to form antiparallel dimers, which aggregate laterally to form anchoring fibrils (10, 11). Because the number of anchoring fibrils (which are restricted in their distribution to the stratifying squamous epithelia) is extremely low even in normal human skin, elucidation of abnormalities in type VII collagen at the protein level is not yet feasible. However, recent cloning of human type VII collagen cDNAs has provided a means to study anchoring fibril involvement in the pathogenesis of EB through genetic linkage analyses (12). Mapping of the type VII collagen gene (COL7A1) has revealed a single locus in the short arm of human chromosome 3 (12). Using a PvuII restriction fragment length polymorphism (RFLP) in the COL7A1 gene, we recently demonstrated linkage between COL7A1 and EB phenotype in a family with EBDD (13). In this study, we examined the genetic linkage of the EB phenotype to the COL7A1 locus and two other polymorphic markers on the short arm of chromosome 3 in three families with EBDD, demonstrating abnormal anchoring fibrils. The segregation analyses, which defined the genetic map locations of EBDD and COL7A1 on chromosome 3, provided evidence for strong linkage between COL7A1 and the EBDD phenotype, suggesting that COL7A1 harbors the mutation in these three families with EBDD.

\section{Methods}

Clinical. Three Finnish pedigrees with EBDD were subjected to study. One of them (FEB33) consisted of 13 affected and 16 unaffected living individuals in two generations (Fig. $1 A$ ). The inheritance of EBDD in this family was consistent with autosomal dominant pattern with complete penetrance. The diagnosis of EBDD (the Cockayne-Touraine type) was made on the basis of clinical observations, including marked tendency for blistering and erosions noted at birth or shortly thereafter. The erosions healed with extensive scarring, and the affected patients 


\section{A. FEB 33}

I

II

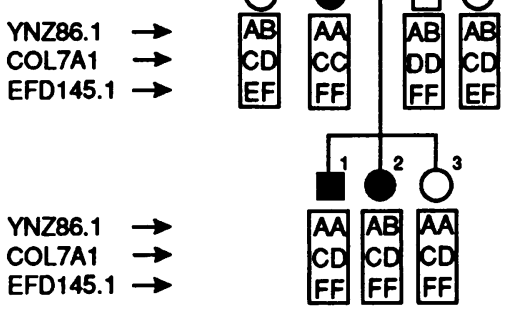

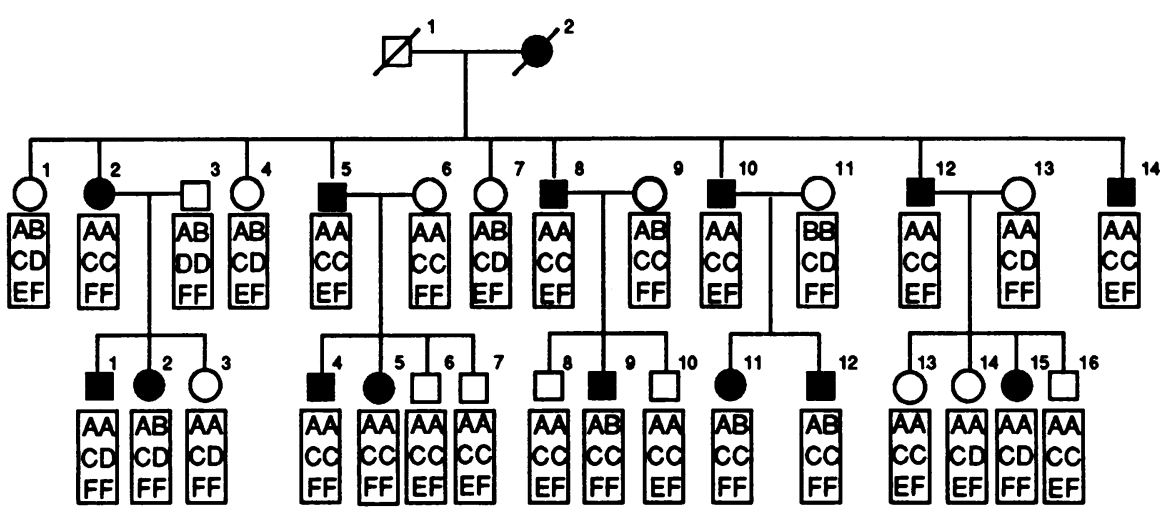

B. FEB 37

I

YNZ86.1 $\rightarrow$ COL7A1 $\rightarrow$ EFD145.1 $\rightarrow$

II

YNZ86.1 $\rightarrow$ EFD145.1 $\rightarrow$

III

\section{FEB 32}

I
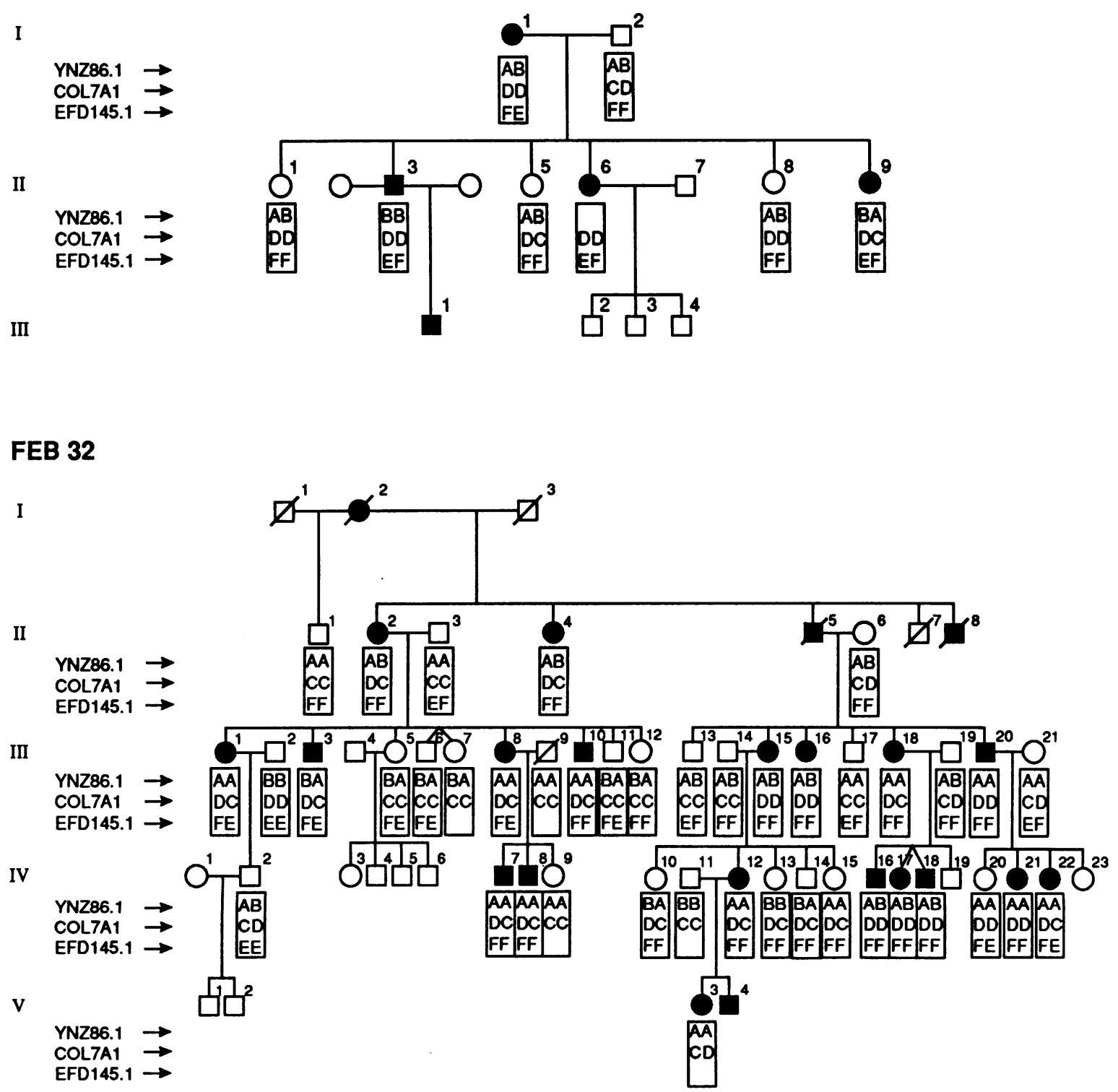

Figure 1. Segregation of D3S30, COL7A1, and D3S32 loci in three kindreds with EBDD. The alleles at each locus are indicated under the symbol for each individual. The alleles are grouped according to most likely haplotypes: YNZ86.1, A, B; COL7A1, C, D; EFD145.1, E, F. 
had characteristic nail dystrophy. Another family (FEB37) with 5 affected and 10 unaffected members was also studied (Fig. $1 \mathrm{~B}$ ). The clinical features were similar to those found in FEB33, and the diagnosis of EBDD was confirmed by histopathology. The inheritance was also consistent with an autosomal dominant pattern. The third family (FEB32), with an EBDD mutation previously shown to be linked to COL7A1 locus (13), was also examined with additional RFLPs on the chromosome 3p (Fig. $1 C$ ).

Skin biopsy specimens were obtained for routine histopathological examination by hematoxylin-eosin stain, for indirect immunofluorescence with a monoclonal anti-type VII collagen antibody $\mathrm{L}_{3} \mathrm{D}$ (14), and for diagnostic electron microscopy.

Southern analyses. DNA was isolated from peripheral blood leukocytes and subjected to digestions with restriction enzymes. The Southern analyses were performed as described previously (15). The DNA probes used for hybridizations of the Southern blots are described in Table I. The two-point linkage data were analyzed by the LIPED program (16), and the multipoint linkage analyses were performed with the LINKAGE programs (17).

\section{Results}

Demonstration of type VII collagen and anchoring fibril defects in families with EBDD. A large kindred (FEB33) with EBDD with 13 affected and 16 unaffected family members was examined (Fig. $1 \mathrm{~A}$ ). Routine histopathology of the apparently normal skin into which mild trauma was applied revealed subepidermal blister formation, apparently within the upper dermis (Fig. 2 A). Indirect immunofluorescence of the unaffected perilesional skin with a MAb $\mathrm{L}_{3} \mathrm{D}$ demonstrated the presence of type VII collagen epitopes in the dermal-epidermal junction (Fig. $2 \mathrm{~B}$ ). However, the staining pattern was weak, irregular, and discontinuous compared with the staining pattern of control skin from an unrelated healthy individual examined in parallel (Fig. $2 C$ ), suggesting alterations in type VII collagen. Diagnostic transmission electron microscopy of the clinically unaffected skin from a patient in this family revealed paucity of anchoring fibrils, and several areas along the basement membrane zone were completely devoid of recognizable anchoring fibrils (Fig. 3, $A-C$ ). The relatively few anchoring fibrils present were largely hypoplastic and demonstrated a markedly reduced diameter (Fig. $3 A$ ) compared with healthy control skin from a normal person (Fig. $3 \mathrm{D}$ ). Thus, the anchoring fibrils were abnormal both in morphology and in numerical density in the skin of affected individuals in this family. Similar histopathological and electron microscopic observations were made in the two other families examined in this study (results not shown).
Thus, COL7A1 could serve as a candidate gene in these families with EBDD.

Genetic linkage of EBDD and COL7A1 loci. The families with EBDD were then analyzed for segregation of RFLPs at the COL7A1 locus and nearby on the short arm of human chromosome 3 (12, 18-20; Table I). Since type VII collagen is the major component of anchoring fibrils (11), COL7A1 was first tested as a candidate gene in the FEB33 family by using a twoallele PvuII RFLP, which we have previously shown to have an allelic frequency of $0.65 / 0.35$ in a normal population with the polymorphism information content of 0.35 (13). Southern hybridizations of genomic DNA revealed cosegregation of the PvuII polymorphism with the EB phenotype in pedigree FEB33 (Fig. $1 A$ ), with a peak logarithm of odds (LOD) score of 2.10 at $\hat{\theta}=0$ (Table II).

Cytogenetic mapping has recently localized COL7A1 to 3p21 (12). Further evidence for linkage of the EBDD mutation to this region of chromosome 3 was obtained with analysis of two anonymous markers (see Table I). Specifically, the probe pYNZ86 (D3S30) yielded $\hat{Z}=2.1$ at $\hat{\theta}=0$, and the probe pEFD145.1 (D3S32) gave a maximum LOD score of 3.74 at $\hat{\theta}=0$.

RFLP analysis indicated the same chromosome 3 map location for the EBDD mutations in two additional families. In family FEB37 (Fig. 1 B), the COL7A1 and D3S30 RFLPs were uninformative for linkage to EBDD, but D3S32 cosegregated with the disease, with a maximum LOD score of 1.51 at $\hat{\theta}=0$ (Table II). The third family (FEB32, Fig. $1 C$ ), in which we have previously demonstrated linkage of EBDD and COL7A1 (13), was also analyzed for segregation of D3S30 and D3S32. The results from FEB32 also supported linkage of EBDD to the chromosome $3 \mathrm{p}$ markers. The $\hat{Z}$ for linkage of EBDD to D3S32 was 2.27 at $\hat{\theta}=0$, and to D3S30, $\hat{Z}=0.88$ at $\hat{\theta}=0.17$ (Table II). With the addition of new family members, the maximum $\hat{Z}$ for COL7A1 and EBDD in the family is now 6.66 at $\hat{\theta}=0$.

The combined LOD score from the three families for linkage of EBDD to COL7A1 locus was 8.77 at $\hat{\theta}=0$. Multipoint analysis with the LINKAGE program placed the EBDD locus closer to D3S32 than to D3S30, with maximum likelihood ( $\hat{Z}$ $=8.2$ ) at D3S32 (Fig. 4). Thus, the linkage data indicate a single locus on chromosome $3 p$ as the site of the EBDD mutation in all three of these families. The absence of recombinants between the COL7A1 and EBDD loci and the demonstrated anchoring fibril defects in the affected individuals strongly suggest that COL7Al harbors the underlying mutation in these families.

Table I. RFLPs in Chromosome 3 Loci Utilized for Linkage Analysis

\begin{tabular}{|c|c|c|c|c|c|c|}
\hline \multirow[b]{2}{*}{$\begin{array}{l}\text { Locus } \\
\text { symbol }\end{array}$} & \multirow{2}{*}{$\begin{array}{c}\text { Map } \\
\text { location }\end{array}$} & \multirow{2}{*}{$\begin{array}{l}\text { DNA } \\
\text { probe }\end{array}$} & \multicolumn{3}{|c|}{ Allele } & \multirow[b]{2}{*}{ Reference } \\
\hline & & & Enzyme & Symbol & Size & \\
\hline & & & & & $k b$ & \\
\hline \multirow{2}{*}{ D3S30 } & $3 p$ & pYNZ86.1 & MspI & A & 2.0 & Nakamura et al. (19) \\
\hline & & & & B & 1.9 & \\
\hline \multirow[t]{2}{*}{ COL7A1 } & $3 \mathrm{p} 21$ & K-131 & PvulI & $\mathrm{C}$ & 3.6 & Parente et al. (12) \\
\hline & & & & D & $1.9,1.7$ & \\
\hline \multirow[t]{2}{*}{ D3S32 } & $3 p$ & pEFD145.1 & RsaI & $\mathbf{E}$ & 2.4 & Fujimoto et al. (20) \\
\hline & & & & $\mathbf{F}$ & $1.4,1.0$ & \\
\hline
\end{tabular}



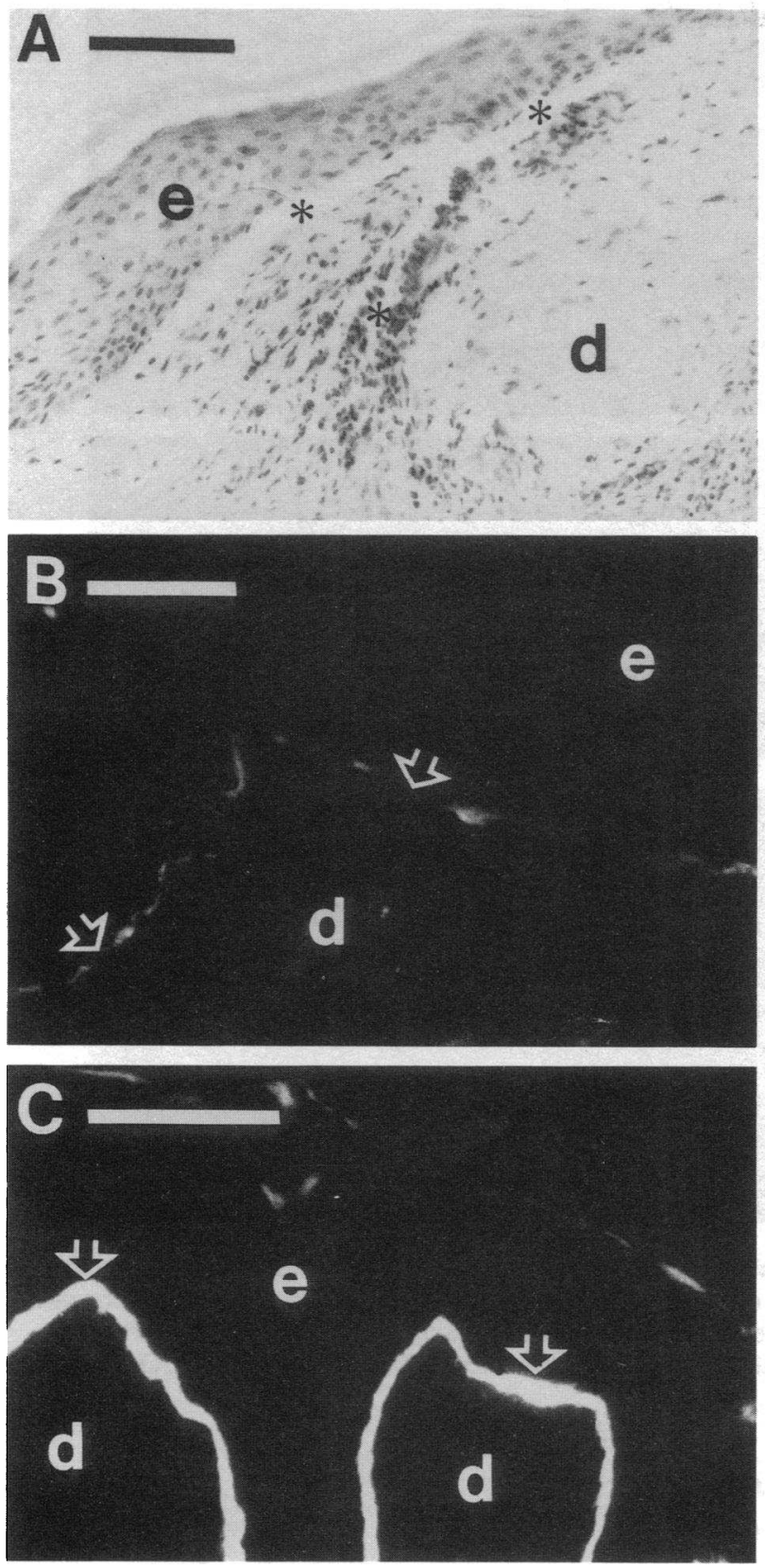

Figure 2. Histopathology and immunofluorescence with anti-type VII collagen antibody. $(A)$ Histopathology of the perilesional skin into which mild trauma was applied demonstrates the presence of a subepidermal blister formation (*). (B) Indirect immunofluorescence with a $\mathrm{MAb}\left(\mathrm{L}_{3} \mathrm{D}\right)$ recognizing type VII collagen epitopes reveals weak and irregular staining in the unaffected skin of the patient with EBDD from kindred FEB33. Note discontinuities in the staining pattern in the affected skin (arrows) compared with the strong linear staining at the cutaneous basement membrane zone of control skin $(C)$. e, epidermis; d, dermis. Bars, $100 \mu \mathrm{m}$.

\section{Discussion}

In this study, we have demonstrated strong genetic linkage of the EB phenotype and the COL7A1 locus on chromosome 3p in three families with the dominant dystrophic form of the disease. The diagnosis of EBDD in these families was based on characteristic clinical presentation and was confirmed histologi- cally by the presence of subepidermal blisters and by electron microscopic demonstration of abnormal anchoring fibrils.

Abnormalities in the anchoring fibrils and the paucity of type VII collagen have been demonstrated in the dystrophic forms of EB (4-6), and the anchoring fibrils and type VII collagen are absent in some cases with severe recessive dystrophic EB (7). Previous cell biological investigations concerning the pathogenesis of recessive dystrophic EB have centered around proteolytic degradation of the anchoring fibrils (21). It has been demonstrated that type VII collagen can be degraded by the interstitial (fibroblast) collagenase (22); and it has been suggested that enhanced levels of collagenase and/or other matrix metalloproteinases, such as stromelysin (MMP-3), cause degradation of anchoring fibrils and result in the clinical phenotype (23-25). However, our present results raise the possibility that structural and/or biosynthetic abnormalities in COL7A1 may be the primary genetic defect in patients with recessive dystrophic EB. In support of this interpretation is a recent demonstration that the collagenase locus on chromosome 11 was excluded as the candidate gene in a family with recessive dystrophic EB by genetic linkage analysis (26). These observations raise at least the possibility of genetic heterogeneity of recessive dystrophic EB.

As indicated above, EB is a group of heritable diseases, and different kinds of mutations are expected to be the underlying cause of clinically distinct phenotypes (27). Previous linkage analyses have suggested the presence of the mutated locus on the long arm of chromosome 1 in some families with the simplex form of EB (EBS) $(28,29)$. Furthermore, a strong genetic linkage to the GPT locus on chromosome 8 has been demonstrated in a family with the rare Ogna variant of EBS (30). In these cases, no candidate gene has been identified, however, and nidogen at the chromosomal locus 1q43 (31) has been excluded as the candidate gene in a family with EBS linked to chromosome 1q (32). More recently, we (33) and others (34) demonstrated a strong genetic linkage of EBS to epidermal keratin gene clusters either on chromosome $12 \mathrm{q}$ or $17 \mathrm{q}$, suggesting that keratin mutations may underlie some forms of EBS. This interpretation is supported by recent immunocytochemical demonstrations of altered keratin gene expression in patients with EBS (35). Furthermore, development of transgenic mice that expressed truncated human keratin 14 resulted in a phenotype reminiscent of the human EBS (36).

It is conceivable that a structural mutation in COL7A1 interferes with the biosynthesis, secretion, or processing of the molecules, manifesting at the supramolecular level as abnormal anchoring fibrils. Thus, COL7A1 defects could be similar to those identified in other collagen genes associated with heritable disorders of connective tissue (37). For example, a large number of mutations have been identified in type I collagen genes in patients manifesting with osteogenesis imperfecta (38); defects in the type II collagen gene, primarily expressed in the cartilaginous tissues, have been identified in patients with chondrodystrophies (39); and type III collagen defects are the likely cause of vascular and cutaneous fragility in patients with the Ehlers-Danlos syndrome type IV (40). Defects in $\alpha 5$ (IV) chain of type IV collagen, which is predominantly expressed in the kidney basement membranes, are the major cause of $\mathrm{Al}$ port's syndrome (41). Thus, genetic mutations in type VII collagen, which is expressed exclusively in the stratified squamous epithelia, could well explain the manifestations of EBDD in the skin and the mucous membranes. 

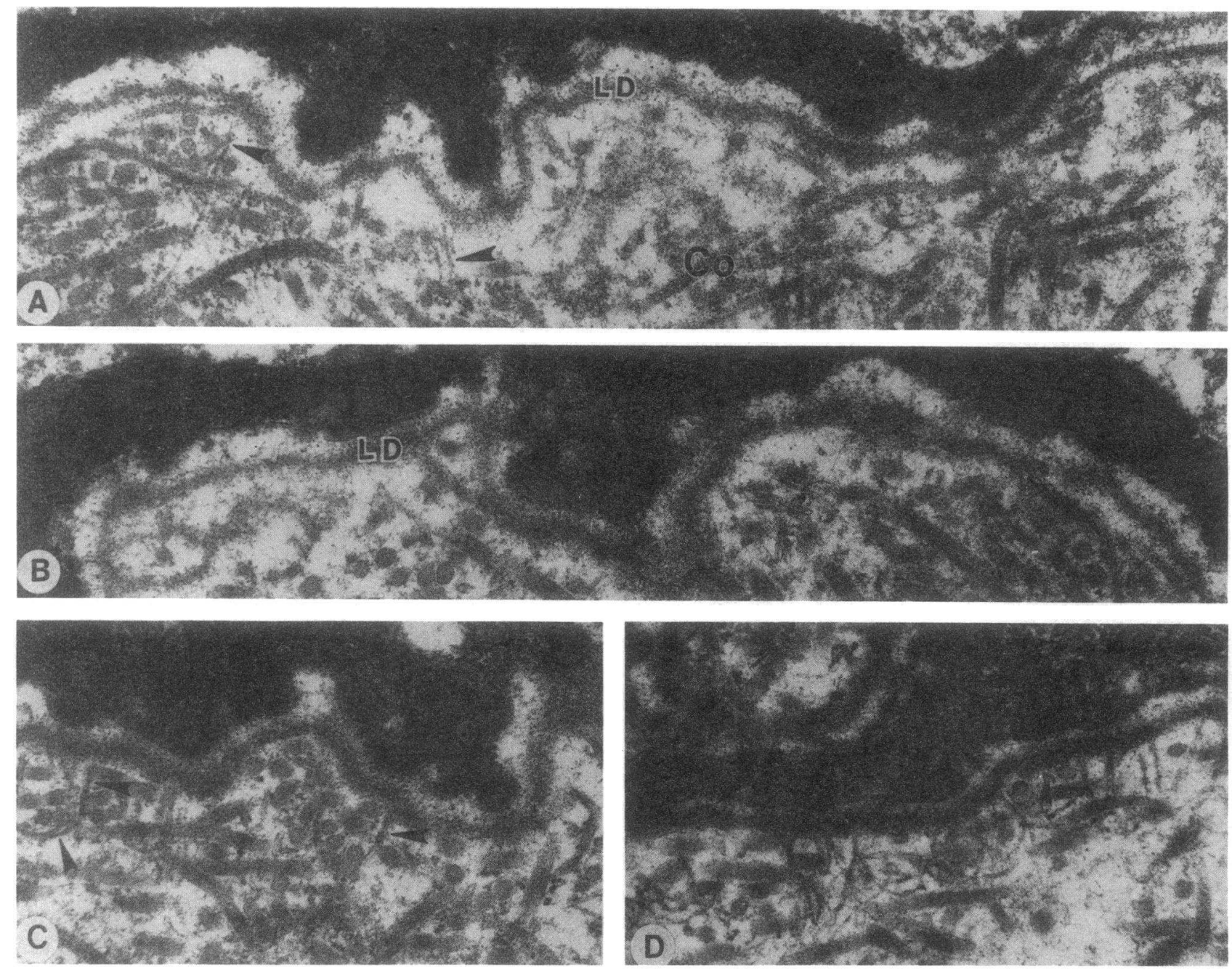

Figure 3. Ultrastructural demonstration of anchoring fibril abnormalities in the skin of a patient from pedigree FEB33. These electron micrographs are representative of three different regions of dermoepidermal junction and show paucity of anchoring fibrils $(A$ and $B)$ and the presence of only a few fibrils $(C)$. In the most affected areas $(A$ and $C,>)$ the fibrils appear thin and rudimentary compared with normal control skin from a healthy individual $(D)$. As expected, both the lamina densa $(L D)$ and the interstitial collagen fibers $(C o)$ are unremarkable. Original magnifications: $A$ and $B, \times 43,500 ; C$ and $D, \times 47,500$.

The possibility of a secretion defect in type VII collagen has been suggested by recent observations that type VII collagen epitopes accumulate within the basal keratinocytes in some individuals with either the dominant dystrophic or the recessive dystrophic form of EB $(42,43)$. Recent studies have also indicated that both epidermal keratinocytes and dermal fibroblasts are capable of expressing COL7A1 in culture, but the basal keratinocytes appear to be the primary cell type responsible for synthesis of type VII collagen during fetal skin development $(44,45)$. Specifically, in situ hybridizations of human skin from fetus of $19 \mathrm{wk}$ gestation revealed an abundance of type VII collagen mRNAs in the basal keratinocytes adjacent to the developing basement membrane zone, whereas dermal fibroblasts were devoid of type VII collagen mRNAs (45). Furthermore, immunofluorescence studies of reconstituted human skin equivalent have suggested that epidermal keratinocytes are the primary source of type VII collagen in anchoring fibrils (44). Thus, by analogy with other heritable disorders of connective tissue, fibroblasts or keratinocytes cultured from patients with EBDD should provide a system to identify the precise mutations and to study the phenotype at the molecular level. Such information will be helpful toward accurate diagno-

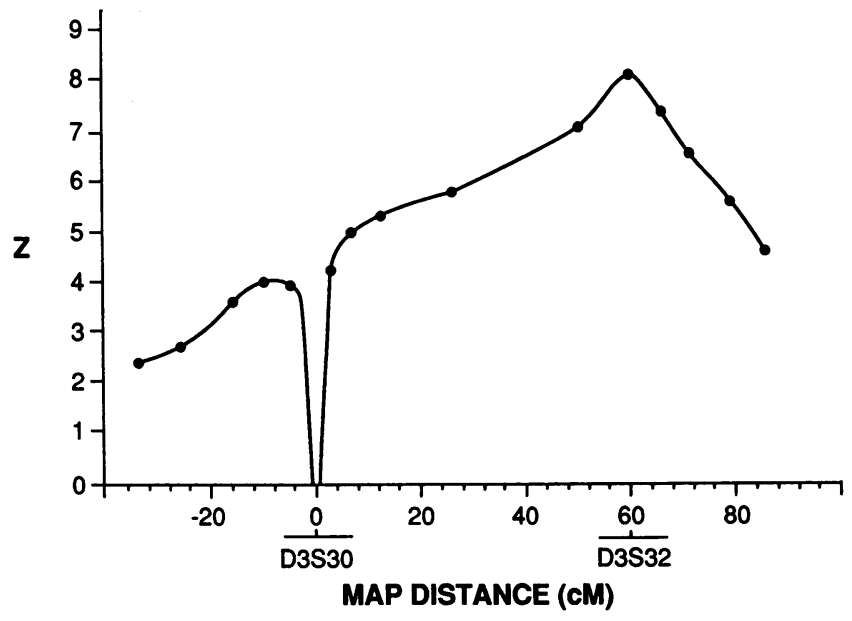

Figure 4. Multipoint analysis of the linkage of EBDD locus to D3S30 and D3S32 on the short arm of chromosome 3. Male genetic map distances (in $\mathrm{cM}$ ) from D3S30 are shown. The sex-specific map distances between D3S30 and D3S32 were reported by Leppert et al.

(18). The vertical axis represents the multipoint LOD score ( $(\hat{Z})$ for EBDD calculated with the LINKMAP program (17). 
Table II. Maximum Two-Point LOD Scores $(\hat{Z})$ for Linkage of Chromosome 3 Loci and EBDD

\begin{tabular}{|c|c|c|}
\hline Loci & $\hat{\theta}$ & $\hat{\mathbf{z}}$ \\
\hline \multicolumn{3}{|c|}{ COL7A1 vs. EBDD } \\
\hline FEB33 & 0 & 2.10 \\
\hline FEB37 & NI & NI \\
\hline FEB32 & 0 & 6.66 \\
\hline Combined & 0 & 8.77 \\
\hline \multicolumn{3}{|c|}{ D3S30 vs. EBDD } \\
\hline FEB33 & 0 & 2.10 \\
\hline FEB37 & $\mathrm{NI}$ & NI \\
\hline FEB32 & 0.17 & 0.88 \\
\hline Combined & 0.10 & 2.45 \\
\hline \multicolumn{3}{|c|}{ D3S32 vs. EBDD } \\
\hline FEB33 & 0 & 3.74 \\
\hline FEB37 & 0 & 1.51 \\
\hline FEB32 & 0 & 2.27 \\
\hline Combined & 0 & 8.11 \\
\hline \multicolumn{3}{|c|}{ D3S30 vs. COL7A1 } \\
\hline FEB33 & 0 & 2.11 \\
\hline FEB37 & $\mathrm{NI}$ & NI \\
\hline FEB32 & 0.17 & 0.77 \\
\hline Combined & 0.11 & 2.32 \\
\hline \multicolumn{3}{|c|}{ D3S32 vs. COL7A1 } \\
\hline FEB33 & 0.50 & 0 \\
\hline FEB37 & NI & NI \\
\hline FEB32 & 0.11 & 0.82 \\
\hline Combined & 0.12 & 0.66 \\
\hline
\end{tabular}

LOD scores were calculated for assumed values of recombination fractions $(\theta)$ by the LIPED program (16). $\hat{\theta}$ and $\hat{Z}$ are maximum likelihood values. NI, not informative.

sis, both pre- and postnatal, in these families, and it would potentially allow identification of subtypes of EBDD at the molecular level. Identification of such mutations might also allow formulation of rational pharmacological approaches to counteract the disease activity, perhaps using approaches that would allow enhanced expression of the normal allele. In this regard, it is of interest that transforming growth factor- $\beta$ is able to significantly enhance COL7A1 expression in cultured keratinocytes (46).

\section{Acknowledgments}

We thank Debra Pawlicki for secretarial help and Bodil Tums for technical assistance. Drs. Matti Kero and Jaakko Karvonen participated in clinical evaluation of the patients. We also thank Dr. Laird Jackson for his support and encouragement.

This study was supported in part by the U. S. Public Health Service, National Institutes of Health grants R01-CA-39481, RO1-CA-47282, PO1-AR-38923, and T32-AR-07869; the Dermatology Foundation; the Paulo Foundation; and the Ella and Georg Ehrnrooth Foundation. M. Ryynänen was a postdoctoral fellow supported by the Division of Medical Genetics, Jefferson Medical College; R. V. Iozzo was recipient of a Faculty Research Award from the American Cancer Society (FRA376); and S. Sollberg was supported by the "Deutsche Forschungsgemeinschaft" grant So239/1-1.

\section{References}

1. Kero, M. 1984. Epidermolysis bullosa in Finland: clinical features, morphology and relation to collagen metabolism. Acta Dermato-Venereol. Suppl. 110:1-51.
2. Gedde-Dahl, T., Jr. 1981. Sixteen types of epidermolysis bullosa. On the clinical discrimination, therapy and prenatal diagnosis. Acta Dermato-Venereol. Suppl. 95:74-78.

3. Fine, J.-D., E. A. Bauer, R. A. Briggaman, D. M. Carter, R. A. J. Eady, N. B. Esterly, K. A. Holbrook, S. Hurwitz, L. Johnson, A. Lin, et al. 1991. Revised clinical and laboratory criteria for subtypes in inherited epidermolysis bullosa: consensus report by the subcommittee on Diagnosis and Classification of the National Epidermolysis Bullosa Registry. J. Am. Acad. Dermatol. 24:119-135.

4. Ghadially, F. N. 1988. Ultrastructural Pathology of the Cell and Matrix Vol. 2. 3rd ed. Butterworths, London. 1228-1230.

5. Tidman, M. J., and R. A. J. Eady. 1985. Evaluation of anchoring fibrils and other components of the dermal-epidermal junction in dystrophic epidermolysis bullosa by a quantitative ultrastructural technique. J. Invest. Dermatol. 84:374377.

6. Briggaman, R. A. 1985 . Is there any specificity to defects of anchoring fibrils in epidermolysis bullosa dystrophica, and what does this mean in terms of pathogenesis? J. Invest. Dermatol. 84:371-373.

7. Bruckner-Tuderman, L., Y. Mitsuhashi, U. W. Schnyder, and P. Bruckner. 1989. Anchoring fibrils and type VII collagen are absent from skin in severe recessive dystrophic epidermolysis. J. Invest. Dermatol. 93:3-9.

8. Sakai, L. Y., D. R. Keene, N. P. Morris, and R. E. Burgeson. 1986. Type VII collagen is a major structural component of anchoring fibrils. J. Cell Biol. 103:1577-1586.

9. Keene, D. R., L. Y. Sakai, G. P. Lunstrum, N. P. Morris, and R. E. Burgeson. 1987. Type VII collagen forms an extended network of anchoring fibrils. $J$. Cell Biol. 104:611-621.

10. Morris, N. P., D. R. Keene, R. W. Glanville, H. Bentz, and R. E. Burgeson. 1986. The tissue form of type VII collagen is an antiparallel dimer. J. Biol. Chem. 261:5638-5644.

11. Burgeson, R. E., G. P. Lunstrum, B. Rokosova, C. S. Rimberg, L. M. Rosenbaum, and D. R. Keene. 1990. The structure and function of type VII collagen. Ann. NY Acad. Sci. 580:32-43.

12. Parente, M. G., L. C. Chung, J. Ryynänen, D. Woodley, K. C. Wynn, E. Bauer, M.-L. Chu, and J. Uitto. 1991. Human type VII collagen: cDNA cloning and chromosomal mapping of the gene. Proc. Natl. Acad. Sci. USA. 88:69316935.

13. Ryynänen, M., R. G. Knowlton, M. G. Parente, L. C. Chung, M.-L. Chu, and J. Uitto. 1991. Human type VII collagen: genetic linkage of the gene (COL7A 1) on chromosome 3 to dominant dystrophic epidermolysis bullosa. Am. J. Hum. Genet. 49:797-803.

14. Paller, A. S., L. L. Queen, D. T. Woodley, E. J. O'Keefe, W. R. Gammon, and R. A. Briggaman. 1985. A mouse monoclonal antibody against a newly discovered basement membrane component, the epidermolysis bullosa acquisita antigen. J. Invest. Dermatol. 82:215-217.

15. Knowlton, R. G., E. J. Weaver, A. F. Stryuk, W. H. Knobloch, R. A. King K. Norris, A. Shamban, J. Uitto, S. A. Jimenez, and D. J. Prockop. 1989. Genetic linkage analysis of hereditary arthro-ophthalmopathy (Strickler syndrome) and the type II procollagen gene. Am. J. Hum. Genet. 45:681-688.

16. Ott, J. 1974. Estimation of the recombination fraction in human pedigrees: efficient computation of the likelihood for human linkage studies. Am. J. Hum. Genet. 26:588-597.

17. Lathrop, G. M., J.-M. Lalouel, C. Julier, and J. Ott. 1984. Strategies for multilocus linkage analysis in humans. Proc. Natl. Acad. Sci. USA. 81:34433446 .

18. Leppert, M., P. O'Connell, Y. Nakamura, P. Cartwright, M. Lathrop, J.-M. Lalouel, and R. White. 1987. Two linkage groups on chromosome 3. Cytogenet. Cell Genet. 46:648.

19. Nakamura, Y., M. Culver, S. Gillian, P. O'Connell, M. Leppert, G. M. Lathrop, J.-M. Lalouel, and R. White. 1987. Isolation and mapping of a polymorphic DNA sequence pYNZ86.1 on chromosome 3 (D3S30). Nucleic Acids Res. 15:10079.

20. Fujimoto, E., Y. Nakamura, J. Gill, P. O'Connell, M. Leppert, G. M. Lathrop, J.-M. Lalouel, and R. White. 1988. Isolation and mapping of a polymorphic DNA sequence (pEFD145) on chromosome 3 (D3S32). Nucleic Acids Res. 16:9357.

21. Bauer, E. A. 1984. Collagenase in recessive dystrophic epidermolysis bullosa. Ann. NY Acad. Sci. 460:311-320.

22. Seltzer, J. L., A. Z. Eisen, E. A. Bauer, N. P. Morris, R. W. Glanville, and R. E. Burgeson. 1989. Cleavage of type VII collagen by interstitial collagenase and type IV collagenase (gelatinase) derived from human skin. J. Biol. Chem. 264:3822-3826.

23. Takamori, K., K. Naito, A. Taneda, and H. Ogawa. 1983. Increased neutral protease and collagenase activity in recessive dystrophic epidermolysis bullosa. Br. J. Dermatol. 108:687-694.

24. Winberg, J.-O., T. Gedde-Dahl, Jr., and E. Bauer. 1989. Collagenase expression in skin fibroblasts from families with recessive dystrophic epidermolysis bullosa. J. Invest. Dermatol. 92:82-85.

25. Sawamura, D., T. Sugawara, I. Hashimoto, L. Bruckner-Tuderman, D. Fujimoto, Y. Okada, N. Utsumi, and H. Shikata. 1991. Increased gene expression of matrix metalloproteinase-3 (stromelysin) in skin fibroblasts from patients with 
severe recessive dystrophic epidermolysis bullosa. Biochem. Biophys. Res. Commun. 174:1003-1008.

26. Hovnanian, A., P. Duquesnoy, S. Amselem, C. Blanchet-Bardon, M. Lathrop, L. Dubertret, and M. Goossens. 1991. Exclusion of linkage between the collagenase gene and generalized recessive dystrophic epidermolysis bullosa phenotype. J. Clin. Invest. 88:1716-1721.

27. McKusick, V. A. 1988. Mendelian Inheritance in Man. Catalogs of Autosomal Dominant, Autosomal Recessive, and X-linked Phenotypes. 8th ed. The Johns Hopkins University Press, Baltimore, pp. 232-234.

28. Mulley, J. C., C. M. Nicholls, D. N. Propert, T. Turner, and G. R. Sutherland. 1984. Genetic linkage analysis of epidermolysis bullosa simplex, Koebner type. Am. J. Med. Genet. 19:573-577.

29. Humphries, M. M., D. Shiels, M. Lawler, G. J. Farrar, P. McWilliam, P. Kenna, D. G. Bradley, E. M. Sharp, M. Young, J. Uitto, et al. 1990. Autosoma dominant simplex epidermolysis bullosa: evidence for linkage to genetic markers on chromosome 1. Genomics. 7:377-381.

30. Olaisen, B., and T. Gedde-Dahl, Jr. 1973. GPT-epidermolysis bullosa simplex (EBS Ogna) linkage in man. Hum. Hered. 23:189-196.

31. Olsen, D. R., T. Nagayoshi, M. Fazio, M.-G. Mattei, E. Passage, D. Weil, R. Timpl, M.-L. Chu, and J. Uitto. 1989. Human nidogen: cDNA cloning, cellular expression, and mapping of the gene to chromosome 1q43. Am. J. Hum. Genet. 44:876-885.

32. Humphries, M., K. Nagayoshi, Y. Sugita, M.-G. Mattei, M.-L. Chu, R. Knowlton, and J. Uitto. 1990. Human nidogen gene: identification of multiple RFLPs and exclusion as candidate gene in a family with epidermolysis bullosa simplex (EBS2) with evidence for linkage to chromosome 1. J. Invest. Dermatol. 95:568-570.

33. Ryynänen, M., R. G. Knowlton, and J. Uitto. 1991. Mapping of epidermolysis bullosa simplex mutation to chromosome 12. Am. J. Hum. Genet. 49:978-984.

34. Bonifas, J. M., A.-L. Rothman, and E. Epstein. 1991. Linkage of epidermolysis bullosa simplex to probes in the region of keratin gene clusters on chromosome 12q and 17q. J. Invest. Dermatol. 96:550. (Abstr.)

35. Ito, M., C. Okuda, N. Shimizu, T. Tazawa, and Y. Sato. 1991. Epidermolysis bullosa simplex (Koebner) is a keratin disorder. Ultrastructural and immunohistochemical study. Arch. Dermatol. 127:367-372.

36. Vassar, R., P. A. Coulombe, L. Degenstein, K. Albers, and E. Fuchs. 1991. Mutant keratin expression in transgenic mice causes marked abnormalities resembling a human genetic skin disease. Cell. 64:365-380.
37. Kuivaniemi, H., G. Tromp, and D. J. Prockop. 1991. Mutations in collagen genes: causes of rare and some common diseases in humans. FASEB (Fed. Am. Soc. Exp. Biol.) J. 5:2052-2060.

38. Prockop, D. J. 1990. Mutations that alter the primary structure of type I collagen: the perils of a system for generating large structures by the principle of nucleated growth. J. Biol. Chem. 265:15349-15352.

39. Vissing, H., M. D'Alessio, B. Lee, F. Ramirez, M. Godfrey, and D. W. Hollister. 1989. Glycine to serine substitution in the triple helical domain of pro $\alpha 1$ (II) collagen results in a lethal perinatal form of short-limbed dwarfism. $J$. Biol. Chem. 264:18265-18267.

40. Kontusaari, S., G. Tromp, H. Kuivaniemi, R. Ladda, and D. J. Prockop. 1990. Inheritance of an RNA splicing mutation $\left(G^{+1 I V s 20}\right)$ in the type III procollagen gene (COL3A1) in a family having aortic aneurysms and easy bruisability: phenotypic overlap between familial arterial aneurysms and Ehlers-Danlos syndrome type IV. Am. J. Hum. Genet. 47:112-120.

41. Zhou, J., D. F. Barker, S. L. Hostikka, M. C. Gregory, C. L. Atkin, and K. Tryggvason. 1991. Single base mutation in $\alpha 5$ (IV) collagen chain gene converting a conserved cysteine to serine in Alport syndrome. Genomics. 9:10-18.

42. Fine, J.-D., Y. Horiguchi, D. H. Stein, N. B. Esterly, and I. M. Leigh. 1990. Intraepidermal type VII collagen, evidence for abnormal intracytoplasmic processing of a major basement membrane protein in rare patients with dominant and possibly localized recessive forms of dystrophic epidermolysis bullosa. J. Am. Acad. Dermatol. 22:188-195.

43. Smith, L. T., and V. P. Sybert. 1990. Intra-epidermal retention of type VII collagen in a patient with recessive dystrophic epidermolysis bullosa. $J$. Invest. Dermatol. 94:261-264.

44. König, A., and L. Bruckner-Tuderman. 1991. Epithelial-mesenchymal interactions enhance expression of collagen VII in vitro. J. Invest. Dermatol. 96:803-808.

45. Ryynänen, J., S. Sollberg, M. G. Parente, L. C. Chung, A. M. Christiano, and J. Uitto. 1992. Type VII collagen gene expression by cultured human cells and in fetal skin. Abundant mRNA and protein levels in epidermal keratinocytes. J. Clin. Invest. 89:163-168.

46. Ryynänen, J., S. Sollberg, D. R. Olsen, and J. Uitto. 1991. Transforming growth factor- $\beta$ up-regulates type VII collagen gene expression in normal and transformed epidermal keratinocytes in culture. Biochem. Biophys. Res. Commun. 180:673-680. 